POS PROCEEDINGS

\title{
Searches for heavy Higgs bosons decaying to light Higgs bosons with a mass of $125 \mathrm{GeV}$
}

\author{
Rebecca Lane* \\ Imperial College London \\ E-mail: rebecca.charlotte.lane@cern.ch \\ On behalf of the CMS Collaboration
}

Searches for Higgs bosons decaying to a pair of Higgs bosons (hh or hA) or for a Higgs boson decaying to $\mathrm{Zh} / \mathrm{ZA}$ are presented. Different analyses involving Higgs boson decays into bottomquarks, tau pairs, and diphotons will be summarized in this talk.

The European Physical Society Conference on High Energy Physics 22-29 July 2015

Vienna, Austria

${ }^{*}$ Speaker. 


\section{Introduction}

The discovery of a $125 \mathrm{GeV}$ Higgs boson at the ATLAS and CMS experiments in 2012 [1,2] has since changed the field of Beyond the standard model (BSM) Higgs searches. The valid options for BSM theories have been limited to those which can incorporate a Higgs boson with a mass close to $125 \mathrm{GeV}$ and coupling properties consistent with those measured at the LHC.

Many such BSM models predict the existence of a heavier resonance decaying into final states containing the $125 \mathrm{GeV}$ Higgs boson. An example of such a process is shown in Figure 1, in which an unknown resonance $\mathrm{X}$ produced via gluon fusion decays into a pair of Higgs bosons, which each decays to a b-anti b quark pair. For a particular choice of model, $X$ could be a radion [3, 4], graviton [5] or a heavier Higgs boson.

Heavier Higgs bosons are predicted by Two Higgs Doublet Model (2HDM) scenarios [6, 7, 8], in which the addition of a second Higgs doublet leads to 5 physical Higgs bosons, 3 of which are neutral - the pseudoscalar A, and the scalar bosons $\mathrm{H}$ and $\mathrm{h}$. Typically such models are expressed in terms of benchmark quantities; commonly used $\operatorname{are} \tan \beta$ and $\alpha$, the ratio of the vacuum expectation values and the mixing angles of the two Higgs doublets respectively. In such a model, either of the scalar $\mathrm{H}$ or $\mathrm{h}$ bosons can be the $125 \mathrm{GeV}$ SM-like Higgs boson discovered at the LHC. The minimally supersymmetric standard model (MSSM) is an example of a 2HDM motivated by supersymmetry [9]. Depending on the choice of mass hierarchy in such a model, the processes $\mathrm{H} \rightarrow \mathrm{hh}, \mathrm{A} \rightarrow \mathrm{Zh} / \mathrm{H}$ or $\mathrm{H} / \mathrm{h} \rightarrow \mathrm{ZA}$ are possible.

In the following proceedings, several analyses are described in which a heavier resonance decays into a final state containing at least one $125 \mathrm{GeV}$ Higgs boson. The analyses all use the $8 \mathrm{TeV}$ dataset taken by CMS during Run 1 of the LHC, which amounts to up to $19.8 \mathrm{fb}^{-1}$ of data.

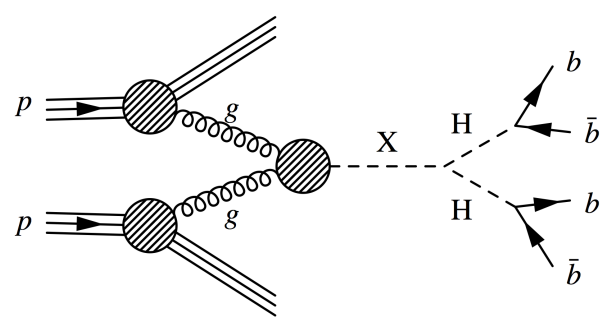

Figure 1: Illustration of an unknown resonance $X$, produced by gluon-fusion, decaying into a pair of Higgs bosons which each decay into a b-anti b quark pair.

\section{Searches in $\mathrm{HH} / \mathrm{hh}$ final states}

\subsection{Search for $\mathbf{X} \rightarrow \mathrm{HH} \rightarrow \gamma \gamma b b$}

The $\mathrm{X} \rightarrow \mathrm{HH} \rightarrow \gamma \gamma \mathrm{bb}$ analysis [10] uses the cut based $\mathrm{H} \rightarrow \gamma \gamma$ selection [11] to select the two photons. Two jets are selected to form the bb candidates, and the events are categorised according to whether 0,1 or 2 of these jets are b-tagged. The $0 \mathrm{~b}$-tagged category has low purity and is used for cross-checks, while the $1 \mathrm{~b}$-tagged (medium purity) and $2 \mathrm{~b}$-tagged (high purity) categories are used as signal regions. 
A range of masses for the candidate particle $\mathrm{X}$ is considered between $260<\mathrm{m}_{\mathrm{X}}<1100 \mathrm{GeV}$. The optimal variable for signal extraction is different for lower and higher mass signal hypotheses. For a candidate particle with $\mathrm{m}_{\mathrm{X}}<400 \mathrm{GeV}$, a fit is made to the diphoton mass $\mathrm{m}_{\gamma \gamma}$, while applying requirements in windows of the 4-body mass $\mathrm{m}_{\gamma \gamma \mathrm{jj}}$. The fit is performed to data using a functional form for the background and signal, and an example can be seen in Figure 2 (left) for the medium purity event category and a signal hypothesis of $m_{X}=300 \mathrm{GeV}$. For a candidate particle with mass $\mathrm{m}_{\mathrm{X}}>400 \mathrm{GeV}$, the 4 body mass $\mathrm{m}_{\gamma \gamma \mathrm{jj}}$, reconstructed using a kinematic fitting method, is used for the fit for signal extraction.

Model independent limits on cross section times branching fraction for the $\mathrm{X} \rightarrow \mathrm{HH} \rightarrow \gamma \gamma \mathrm{bb}$ process are set and compared to predictions from radion and graviton models, as can be seen in Figure 2 (right). The analysis excludes a radion with $\Lambda_{\mathrm{R}}=1 \mathrm{TeV}$ for masses below $0.97 \mathrm{TeV}$ and the RS1 KK-graviton with masses between 340 and $400 \mathrm{GeV}$.
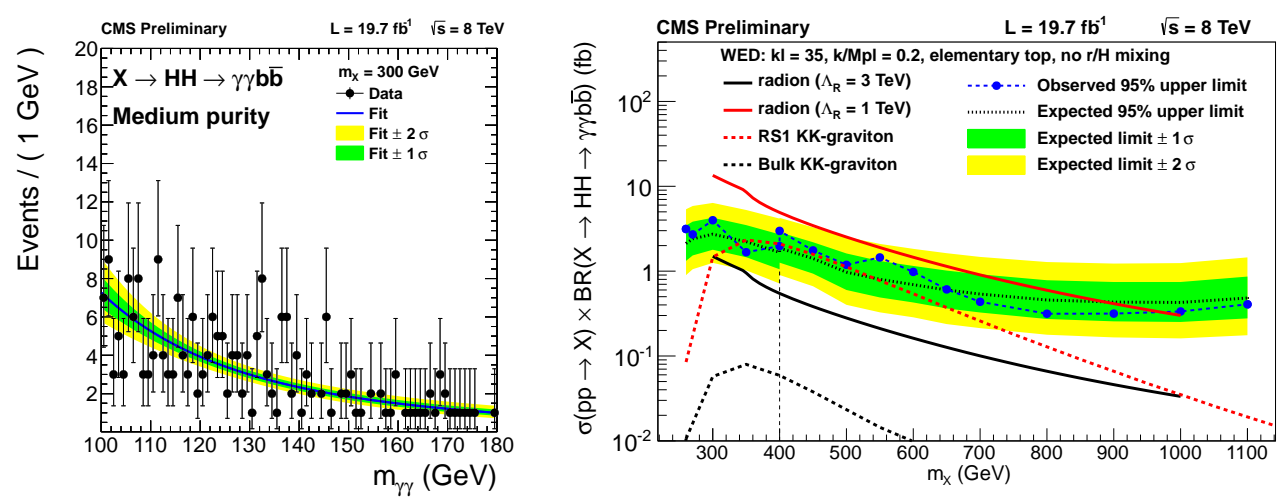

Figure 2: Left: Example of a fit for signal extraction in the medium purity category. The fit is performed to the variable $\mathrm{m}_{\gamma \gamma}$ for a signal hypothesis of $\mathrm{m}_{\mathrm{X}}=300 \mathrm{GeV}$. Right: Expected and observed limit on the cross section times branching fraction for the $\mathrm{X} \rightarrow \mathrm{HH} \rightarrow \gamma \gamma \mathrm{bb}$ process from the combination of all categories. The limit is compared with predictions for choices of radion and graviton models [10].

\subsection{Search for $\mathbf{X} \rightarrow \mathrm{HH} \rightarrow$ bbbb}

This search [12] takes a similar model independent approach considering signal hypothesis masses between $270<\mathrm{m}_{\mathrm{X}}<1100 \mathrm{GeV}$ and comparing to radion and graviton models. This analysis is also separated into lower and higher signal masses for the most optimal signal extraction; for the lower masses $\left(\mathrm{m}_{\mathrm{X}}<450 \mathrm{GeV}\right)$ the candidate jets are paired requiring a combined invariant mass as close as possible to $125 \mathrm{GeV}$, whereas for higher masses $\left(\mathrm{m}_{\mathrm{X}}>450 \mathrm{GeV}\right)$ jets which have the smallest $\Delta R=\sqrt{\Delta \eta^{2}+\Delta \phi^{2}}$ are paired. The model independent limits for the radion and graviton signal hypotheses are shown in Figure 3. The analysis is able to exclude a radion with $\Lambda_{R}=1 \mathrm{TeV}$ for masses between 300 and $1100 \mathrm{GeV}$, and a KK-graviton with masses between 380 and $830 \mathrm{GeV}$.

\subsection{Search for $H \rightarrow h h \rightarrow b b \tau \tau$}

The $\mathrm{H} \rightarrow \mathrm{hh} \rightarrow \mathrm{bb} \tau \tau$ analysis [13] uses the inclusive $\mathrm{H} \rightarrow \tau \tau$ [14] selection to select a candidate $\tau \tau$ pair, in any of the three most sensitive channels e $\tau_{h}, \mu \tau_{h}$ and $\tau_{h} \tau_{h}$, where $\tau_{h}$ indicates 

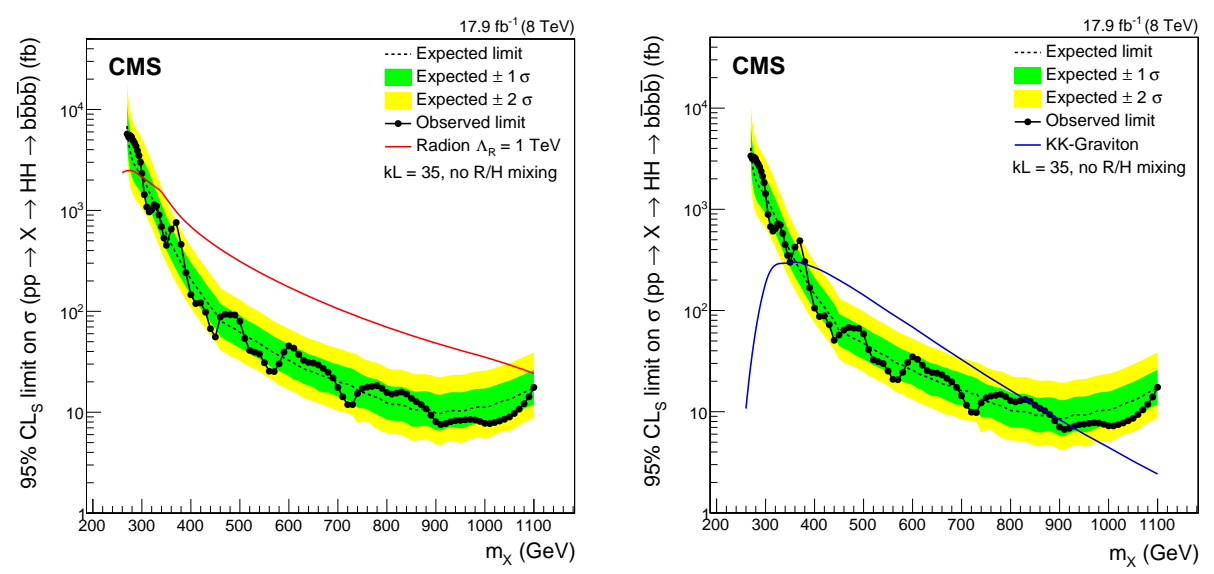

Figure 3: Expected and observed limit on the cross section times branching fraction for the $\mathrm{X} \rightarrow \mathrm{HH} \rightarrow \mathrm{bbbb}$ process for the radion (left) and graviton (right) signal hypotheses [12].

a hadronically decaying tau. Two jets are required to form the candidates from the $\mathrm{h} \rightarrow \mathrm{bb}$ decay, and the events are categorised according to whether 0,1 or 2 of these jets are b-tagged.

A selection is applied on the reconstructed di-tau mass $\mathrm{m}_{\tau \tau}$ and di-jet mass $\mathrm{m}_{\mathrm{jj}}$ in windows around $125 \mathrm{GeV}$, specifically $70<\mathrm{m}_{\mathrm{jj}}<150 \mathrm{GeV}$ and $90<\mathrm{m}_{\tau \tau}<150 \mathrm{GeV}$. The signal extraction is performed to the 4-body mass, reconstructed using a kinematic fit and denoted $\mathrm{m}_{\mathrm{H}}^{\text {kinfit }}$. An example such distribution, for events in the $\mu \tau_{h}$ final state in which one of the jets is b-tagged, is shown in Figure 4 (left).

A model independent limit on the $\mathrm{H} \rightarrow \mathrm{hh} \rightarrow \mathrm{bb} \tau \tau$ process is shown in Figure 4 (right). Model dependent results in 2HDM and MSSM interpretations are produced for this analysis in combination with the $\mathrm{A} \rightarrow \mathrm{Zh} \rightarrow \ell \ell \tau \tau$ analysis described in section 3.2.
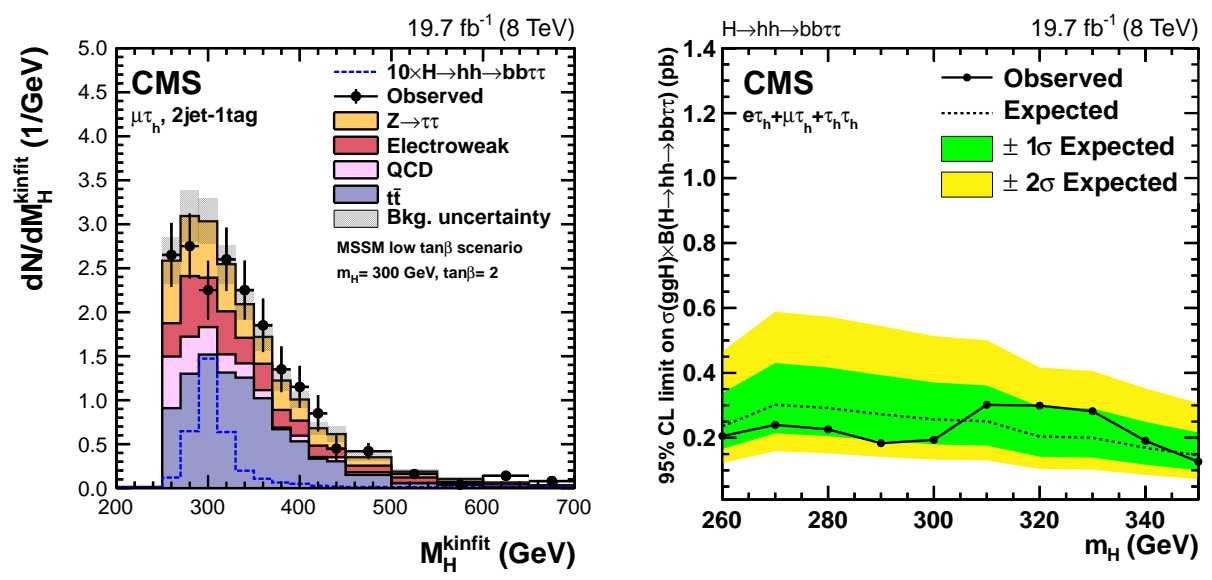

Figure 4: Left: Example 4-body mass distribution as extracted from the kinematic fit. Right: Expected and observed limit on cross section times branching fraction for the $\mathrm{H} \rightarrow \mathrm{hh} \rightarrow \mathrm{bb} \tau \tau$ process for the combination of all channels and categories [13]. 


\section{Searches in $\mathrm{ZH} / \mathrm{Zh} / \mathrm{ZA}$ final states}

\subsection{Search for $\mathrm{A} \rightarrow \mathrm{Zh} \rightarrow \ell \ell \mathrm{bb}$}

For selecting events in a final state containing a $\mathrm{Z}$ boson, the clean signatures from $\mathrm{Z} \rightarrow \ell \ell$ $(\ell=\mathrm{e}, \mu)$ are used. The candidate bb pair for this analysis [15] is selected using two jets, both of which pass the loose b-tagging working point and one of which passes the medium working point. The 4-body mass $m_{\ell \ell b b}$ is reconstructed using a kinematic fitting method. For signal extraction, a 2D fit is performed to the 4-body mass and a BDT discriminator, trained in 3 different ranges of $\mathrm{m}_{\mathrm{A}}$ hypotheses.

The results of this analysis are presented in both model independent and model dependent forms. The model independent limit on cross section times branching fraction for the $\mathrm{A} \rightarrow \mathrm{Zh} \rightarrow$ $\ell \ell$ bb process is shown in Figure 5 (left). Model dependent limits are set in type-I and type-II 2HDM scenarios in which $\mathrm{m}_{\mathrm{H}}$ and $\mathrm{m}_{\mathrm{A}}$ are fixed to particular values. The exclusion is expressed in the $2 \mathrm{D}$ plane of $\tan \beta$ and $\cos (\beta-\alpha)$. An example of such an exclusion, for a type-II $2 \mathrm{HDM}$ with $\mathrm{m}_{\mathrm{A}}=$ $\mathrm{m}_{\mathrm{H}}=300 \mathrm{GeV}$ is shown in Figure 5 (right).
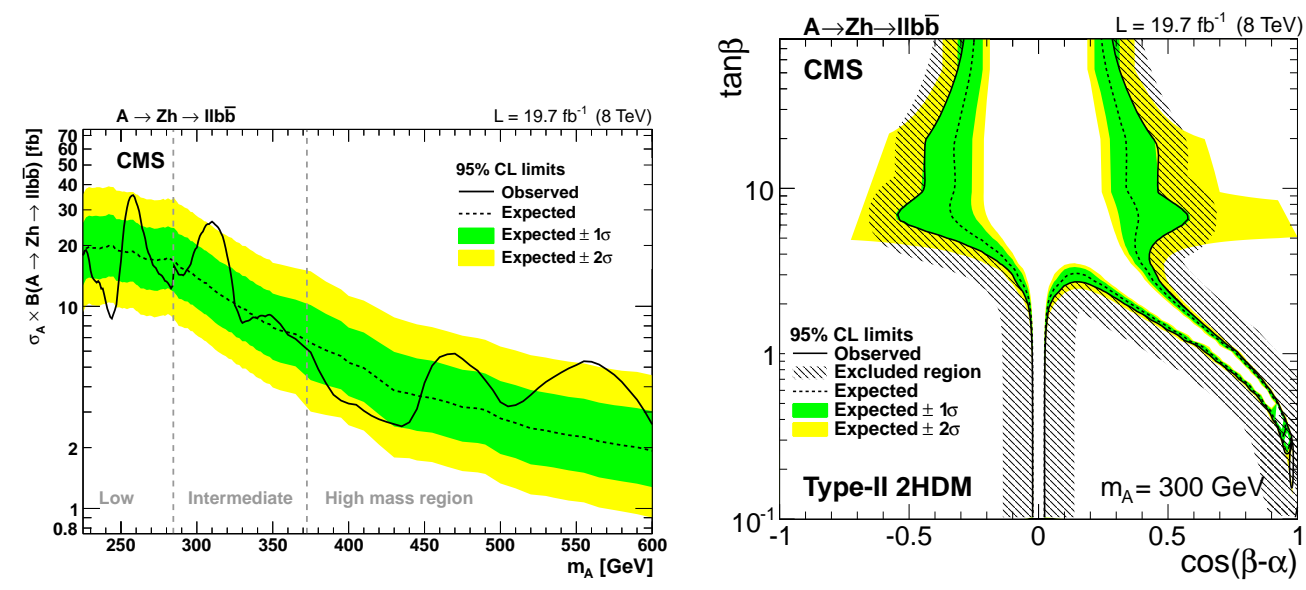

Figure 5: Left: Expected and observed limit on cross section times branching fraction for the $\mathrm{A} \rightarrow \mathrm{Zh} \rightarrow$ $\ell \ell$ bb process. Right: Expected and observed limit in a $2 \mathrm{HDM}$ type-II scenario in which $\mathrm{m}_{\mathrm{A}}=300 \mathrm{GeV}$ [15].

\subsection{Search for $\mathrm{A} \rightarrow \mathrm{Zh} \rightarrow \ell \ell \tau \tau$}

In this final state [13] the same approach is made of selecting $\mathrm{Z} \rightarrow \ell \ell$ events. For the tau pair the 4 most sensitive final states are used e $\tau_{h}, \mu \tau_{h}, \tau_{h} \tau_{h}$, and $\mathrm{e} \mu$ are used. The 4-body mass $\mathrm{m}_{\mathrm{A}}$ is reconstructed by combining the 4 -vectors from the $\mathrm{Z}$ boson and the $\tau \tau$ candidates and is the variable used for signal extraction. An example of one such distribution is shown in Figure 6 (left) for the $\tau_{h} \tau_{h}$ final state using $\mathrm{Z} \rightarrow$ ee decays. A total of 8 different channels are combined for all possible combinations of $\mathrm{Z}$ boson and $\tau \tau$ final states.

The model independent limit on cross section times branching fraction for the $\mathrm{A} \rightarrow \mathrm{Zh} \rightarrow \ell \ell \tau \tau$ process is shown in Figure 6 (right). Model dependent limits are also set in combination with the $\mathrm{H} \rightarrow \mathrm{hh} \rightarrow \mathrm{bb} \tau \tau$ analysis described in section 2.3. These are set in a low-tan $\beta$ appropriate MSSM scenario [16] in Figure 7 (left) and in a type-II 2HDM scenario (the same scenario as shown in the 
previous section) in Figure 7 (right). Note that following the conference further work has produced updated limits, so these figures are modified with respect to those shown in the conference talk.
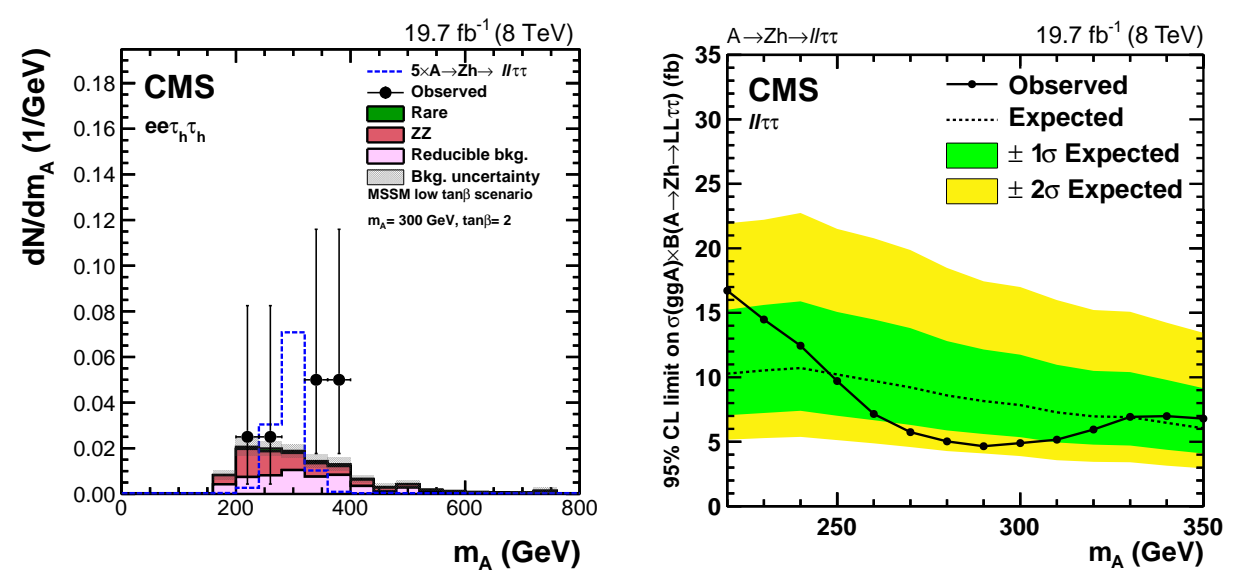

Figure 6: Left: Example 4-body mass distribution for events in the $\tau_{h} \tau_{h}$ channel in which the $\mathrm{Z}$ boson decays to ee. Right: Expected and observed limit on cross section times branching fraction for the $\mathrm{A} \rightarrow$ $\mathrm{Zh} \rightarrow \ell \ell \tau \tau$ process for the combination of all channels and categories [13].
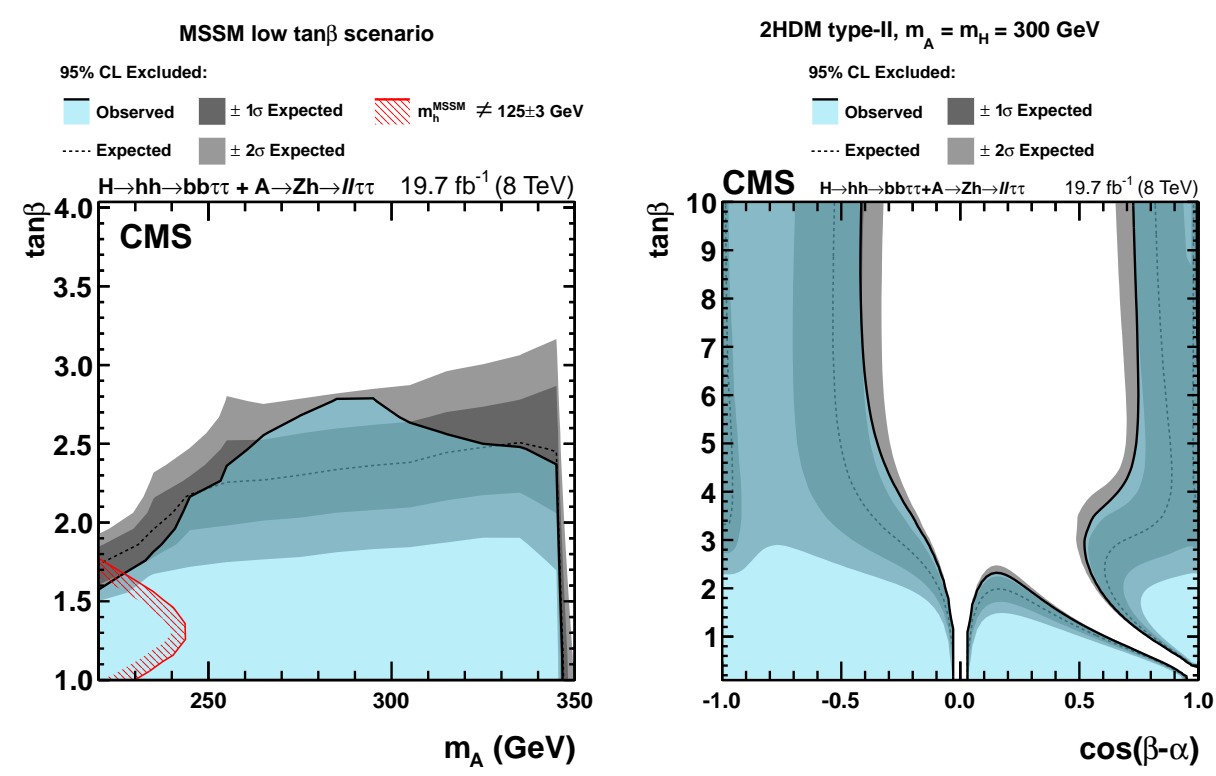

Figure 7: Expected and observed limit in the MSSM low-tan $\beta$ scenario (left) and the type-II 2HDM scenario in which $\mathrm{m}_{\mathrm{A}}=\mathrm{m}_{\mathrm{H}}=300 \mathrm{GeV}$ (right) [13].

Note that a more model independent approach to both $\mathrm{A} \rightarrow \mathrm{Zh}$ analyses, in which both possible mass hierarchies $m_{H}>m_{A}$ and $m_{A}>m_{H}$ are considered, is been illustrated in [17]. Interpretations in $2 \mathrm{HDM}$ scenarios can also be found in this publication. 


\section{References}

[1] ATLAS Collaboration, "Observation of a new particle in the search for the Standard Model Higgs boson with the ATLAS detector at the LHC”, Phys. Lett. B 716 (2012)

doi:tt10.1016/j.physletb.2012.08.020, arXiv:1207.7214.

[2] CMS Collaboration, "Observation of a new boson at a mass of $125 \mathrm{GeV}$ with the CMS experiment at the LHC”, Phys. Lett. B 716 (2012) doi:tt10.1016/j.physletb.2012.08.021, arXiv:1207.7235.

[3] W. D. Goldberger and M. B. Wise, "Modulus Stabilization with Bulk Fields", Phys. Rev. Lett. 83 (Dec, 1999) 4922-4925, doi : tt10.1103/PhysRevLett.83.4922.

[4] C. Csaki, M. Graesser, L. Randall et al., "Cosmology of brane models with radion stabilization", Phys. Rev. D62 (2000) 045015, doi : tt10.1103/PhysRevD.62.045015, arXiv:hep-ph/9911406.

[5] H. Davoudiasl, J. L. Hewett, and T. G. Rizzo, "Phenomenology of the Randall-Sundrum Gauge Hierarchy Model”, Phys. Rev. Lett. 84 (Mar, 2000) 2080-2083, doi : tt10.1103/PhysRevLett.84.2080.

[6] S. L. Glashow and S. Weinberg, "Natural conservation laws for neutral currents", Phys. Rev. D 15 (1977) 1958, doi : tt10.1103/PhysRevD.15.1958.

[7] J. F. Gunion, H. E. Haber, G. L L. Kane et al., “The Higgs Hunter's Guide”, Front. Phys. 80 (2000) 1.

[8] G. Branco, P. Ferreira, L. Lavoura et al., "Theory and phenomenology of two-Higgs-doublet models", Phys. Rept. 516 (2012) 1, doi:tt10.1016/j.physrep.2012.02.002, arXiv:1106.0034.

[9] S. P. Martin, “A Supersymmetry primer”, arXiv:hep-ph/9709356.

[10] CMS Collaboration, "Search for resonant HH production in 2gamma+2b channel", Technical Report CMS-PAS-HIG-13-032, CERN, Geneva, (2014).

[11] CMS Collaboration, "Observation of the diphoton decay of the Higgs boson and measurement of its properties”, Eur. Phys. J. C74 (2014), no. 10, 3076, doi : tt10.1140/epjc/s10052-014-3076-z, arXiv:1407.0558.

[12] CMS Collaboration, "Search for resonant pair production of Higgs bosons decaying to two bottom quark antiquark pairs in proton proton collisions at 8 TeV”, Phys. Lett. B749 (2015) 560-582, doi:tt10.1016/j.physletb.2015.08.047, arXiv:1503.04114.

[13] CMS Collaboration, "Searches for a heavy scalar boson $\mathrm{H}$ decaying to a pair of $125 \mathrm{GeV}$ Higgs bosons hh or for a heavy pseudoscalar boson A decaying to $\mathrm{Zh}$, in the final states with h to tautau", arXiv:1510.01181.

[14] CMS Collaboration, "Search for neutral MSSM Higgs bosons decaying to a pair of tau leptons in pp collisions”, JHEP 10 (2014) 160, doi :tt10.1007/JHEP10(2014)160, arXiv:1408.3316.

[15] CMS Collaboration, "Search for a pseudoscalar boson decaying into a Z boson and the $125 \mathrm{GeV}$ Higgs boson in ${ }^{+} b \bar{b}$ final states", Phys. Lett. B748 (2015) 221-243, doi:tt10.1016/j.physletb.2015.07.010, arXiv:1504.04710.

[16] E. Bagnaschi, F. Frensch, S. Heinemeyer et al., "Benchmark scenarios for low $\tan \beta$ in the MSSM”, Technical Report LHCHXSWG-2015-002, CERN, Geneva, (Aug, 2015).

[17] CMS Collaboration, "Search for H/A decaying into $\mathrm{Z}+\mathrm{A} / \mathrm{H}$, with $\mathrm{Z}$ to $\mathrm{ll}$ and $\mathrm{A} / \mathrm{H}$ to fermion pair", Technical Report CMS-PAS-HIG-15-001, CERN, (2015). 